\title{
Allogenic Stem Cell Transplantation and Total Body Irradiation
}

\author{
Serra KAMER \\ Department of Radiation Oncology, Ege University Faculty of Medicine, İzmir-Turkey
}

\begin{abstract}
SUMMARY
The clinical importance of bone marrow transplantation has increased considerably in recent decades. More than 450 centers for bone marrow transplantation now exist worldwide, performing more than 5000 transplantations per year. The number of transplant centers and patients has increased dramatically in Turkey in the last several years. When transplant donor and recipient are different individuals, hematopoietic graft is known as allogeneic graft. Total body irradiation (TBI) remains an important component of allogeneic hematopoietic stem cell transplant, with the goal of eradicating residual malignant cells and modulating the immune system of the transplant recipient. TBI is especially advantageous in allogeneic stem cell transplantation, since its biological effects can be exerted uniformly without sparing the "sanctuary" sites, such as nervous system or testicles. In this report, the role of TBI in allogeneic stem cell transplantation is reviewed.
\end{abstract}

Keywords: Allogenic stem cell transplantation; bone marrow donor; total body irradiation.

Copyright $\odot$ 2016, Turkish Society for Radiation Oncology

Allogenic stem cell transplantation involves transferring the stem cells from a healthy person (the donor) to a patient (the recipient) following high-intensity chemotherapy and/or irradiation. The donor can be a relative of the patient or a stranger named as unrelated donor. The important thing is that the donor's immune system markers should closely matched to patients. To find a HLA -matched sibling donor is not possible for $70 \%$ of the patients who need immediately an allogenic hematopoietic stem cell transplant.[1] To overcome this many countries developed regional or national bone marrow databases to find a matching donor. In Turkey the Turkkök Project (National Marrow Donor Program) has developed into a large national organization, allowing access to a large database of unrelated donors, where data of more than one million healthy persons stored. This has resulted increased chance of finding a matching donor for patients of all races/ethnicities. The Turkkök Project has also developed programs and approaches that have successfully increased the efficiency of the search process, which has decreased the waiting time to transplant. It is also expected an increase in the feasibility of allogeneic stem cell transplanation and requirement of total body irradiation (TBI) applications in our country with this project.

Allogeneic Stem Cell Transplantation, using human leukocyte antigen (HLA)-matched sibling or unrelated bone marrow donors has been used successfully to treat patients with high-risk or relapsed hematologic malignancies.[1,2] Some solid tumors are under the investigation of allogeneic transplantation (Table 1). Successful bone marrow transplantation with a combination of cyclophosphamide and total body irradiation as a conditioning regimen was first reported in the 1970s.[3] 
Table 1 Tumors which allogenic transplantation using with total body irradiation is performed

Standard treatment

Acute lymphoblastic leukemia, (ALL)

Acute/chronic myelogenous leukaemia (AML/CML)

\section{Under clinical investigation}

Neuroblastoma,

Ewing sarcoma,

High/low grade non-Hodgkin lymphoma,

Multiple myeloma
TBI is an important component of hematopoietic stem cell transplant with the goal of eradicating residual malignant cells and modulating the immune system of the transplant recipient. TBI has several advantages over chemotherapy since its biologic effects can be exerted uniformly throughout the body without sparing of the "sanctuary" sites such as the nervous system or testis, which is evident for many chemotherapy drugs. However, there are always concerns with the use of irradiation regarding the long term sequelae, including cataracts, second malignancies, and developmental problems in pediatric cases specifically. Because of these concerns, chemotherapy regimens omitting total body irradiation have been studied extensively where busulfan replaced TBI.[4,5] Both TBI/Cyclophosphamide or Busulfan/Cyclophosphamide regimens have been accepted standard conditioning regimens since the 1980s. There are a few published papers comparing TBI/Cyclophosphamide versus Busulfan/Cyclophosphamide for stem cell transplantion and their long-term results for different leukaemia types (Table 2). In 2010 , a meta-analysis compared the clinical results of different conditioning regimens for various leukaemias. In this metaanalysis, different studies were evaluated for therapeutic effects of TBI/CY or BU/CY regimens with assessment of engraftment, relapse patterns, complications, and disease-free survival.[6] A total of 3172 patients from 18 trials have been evaluated and TBI/CY regimen was reported to have similar

\begin{tabular}{lccc} 
Table 2 & $\begin{array}{c}\text { The results of TBI containing regimens in acute } \\
\text { lymphoblastic leukemia }\end{array}$ \\
Author & Regimen & Overall Survival \\
\hline Bunin[5] & $\mathrm{BU}$ & $67 \%$ & \\
& $\mathrm{TBI}$ & $47 \%$ & 3 year \\
Davies[4] & $\mathrm{CY} / \mathrm{TBI}$ & $55 \%$ & \\
& $\mathrm{BU} / \mathrm{CY}$ & $40 \%$ & 3 year \\
Park J[15] & $\mathrm{TBI} / \mathrm{CY}$ & $64.3 \%$ & 2 year \\
Kamer - unpublished results & $\mathrm{TBI} / \mathrm{CY}$ & $69 \%$ & 2 year \\
\hline
\end{tabular}

BU: Busulfan; CY: Cyclophosphamide; TBI: Total body irradiation. occurrence of engraftment, acute and chronic graftversus-host disease with $\mathrm{BU} / \mathrm{CY}$, but had higher rates of cataract, interstitial pneumonitis, later growth or developmental problems.[6] BU/CY regimen was related with more severe complications such as veno-occlusive disease of liver, hemorrhagic cystitis, and treatment-related mortality The authors also concluded that different treatment regimens and leukaemia types may affect the complications and outcome.[6] The results of TBI containing regimens in acute lymphoblastic leukemia (ALL) is summarized in Table 2. The ideal conditioning regimen for leukaemia patients undergoing bone marrow transplantation still remains unknown and the results of current ongoing BFM trial will be the answer of this question in the near future.

Numerous different techniques have been developed to deliver TBI.[7-10] The choice of a particular technique varies among institutes and depends onexperience of the institute, the workload of the department, geometry of the treatment room, available infrastructure and equipment, and treatment protocol. In the majority of departments two treatment techniques or combination of both are used in practice. An anteroposterior posteroanterior (AP/PA) technique generally provides a better dose uniformity along the longitudinal body axis, but it is not possible to cover the whole body with a single field and use of multiple neighboring fields is always a challenge. Bilateral TBI (treating from the left and right side of the body) can be more comfortable to the patient if seated on a specially designed TBI Chair (Figure 1) but, presents greater variation in body thickness along the path of the beam. Thus compensators are required to achieve dose uniformity along the body axis.

The aims of designing a treatment technique for an individual department should be as follows: performing TBI within the department's regular schedule, using the best possible technique requiring a short treatment time, providing a comfortable positioning to patients, improving and simplifying the lung shielding system to provide reproducible positioning of the patients. Therefore, the ideal treatment technique comprises the 


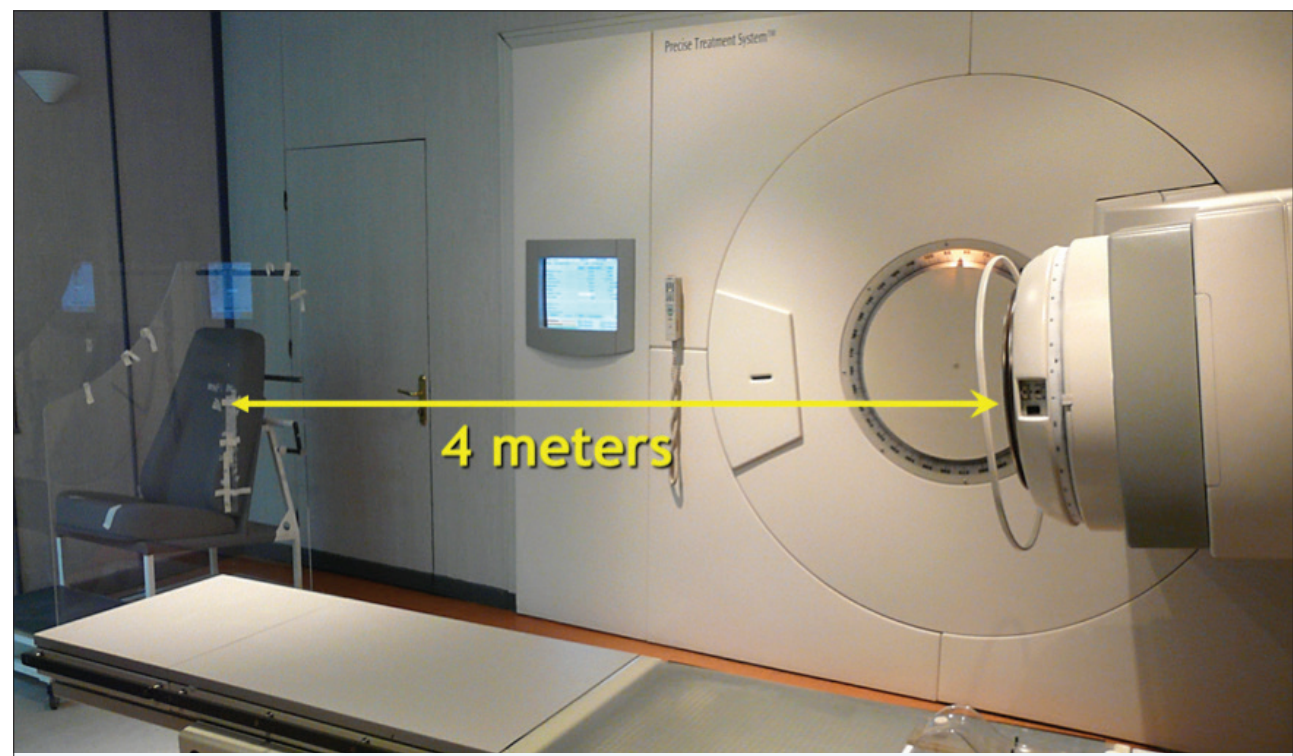

Fig. 1. TBI set-up at the Ege University Hospital. Bilateral irradiation is used with an SSD of 4 meters. Patient is seated on a specially designed chair. Plexiglas beam screens are used to increase the surface dose. Reference dose is measured by $0.6 \mathrm{cc}$ ion chamber taped on the plexiglas screen.

requirements of dose homogeneity, lung sparing and dose prescription, accuracy of treatment, reproducibility and reliability of treatment set-up, comfort for patient and staff. The technique of TBI has evolved in parallel with an increase in the knowledge of the biologic response to ionizing radiation and improvements in radiation dosimetry and treatment delivery. Recently, new advanced techniques using helical tomotherapy and VMAT (volumetric modulated arc therapy) were implemented for TBI.[9] VMAT dose distribution was better than conformal techniques but total treatment time was unacceptably longer (coach time was 2 hours per day) for daily use especially for small kids. Longer treatment times was also reported with helical tomotherapy.[7] Another disadvantage of helical tomotherapy is the limited treatment length of the longitudinal axis, allowing a maximum PTV length of $145 \mathrm{~cm}$. Patients exceeding $145 \mathrm{~cm}$ body length require another complex plan for lower part of the body, which extends the total treatment time further.

The best total radiotherapy dose and dose rate are other unanswered questions in clinical practice. Several authors tried to find a relationship between the total dose of TBI and treatment outcome. Although some of them reported a higher overall survival with increasing TBI dose,[10] others reported opposite.[11] Vriesendorp et al.[12] reported that the results of TBI is related with dose, fraction size and endpoint selection and that different TBI procedures could not be compared without radiobiological "normalization". This normalization will also be different for various endpoints. Relapse rates were significantly lower in the TBI schedules using higher doses but disease-free survival and treatment-related mortality were significantly different in various trials.[13]

Acute and especially long term side effects are the major concern for TBI protocols.[14] Most centres use fractionated TBI to reduce acute side effects such as nausea and vomiting, and late effects such as cataracts. Shielding of the lungs to keep the total lung doses under $10 \mathrm{~Gy}$ is also widely used to prevent severe radiation pneumonitis. In Europe, most centres do not irradiate children below the age of 2 years due to the harmful effects on the developing brain. The biggest risks for children who received TBI are the secondary malignancies, growth retardation (especially if irradiated below 10 years) and infertility (most common after irradiation during or after puberty).

To date, it has not been shown that TBI in the conditioning regimen for childhood ALL can be replaced by chemotherapy. Davies et al. compared outcomes of HLA-identical sibling transplants for ALL in children who received cyclophosphamide CY/TBI $(n=451)$ versus those who received $\mathrm{Bu} / \mathrm{CY}(\mathrm{n}=176)$ for pre-transplant conditioning. The 3-year probabilities of survival were $55 \%$ with $\mathrm{TBI} / \mathrm{CY}$ and $40 \%$ (95\% CI $32 \%$ to $48 \%$ ) 
with $\mathrm{Bu} / \mathrm{CY}$ (univariate $\mathrm{p}=.003$ ). In a multivariate analysis, the risks of relapse were similar in the two groups (relative risk [RR], 1.30 for $\mathrm{Bu} / \mathrm{CY} \mathrm{v} \mathrm{CY} / \mathrm{TBI}$; $\mathrm{p}=.1$ ). Treatment related mortality was higher in the $\mathrm{Bu} / \mathrm{CY}$ group (RR, 1.68; $\mathrm{p}=.012$ ). Death and treatment failure (relapse or death, inverse of leukaemia-free survival (LFS)) were more frequent in the $\mathrm{Bu} / \mathrm{CY}$ group (RR, 1. 39; $\mathrm{p}=.017$ for death; $\mathrm{RR}, 1.42 ; \mathrm{p}=.006$ for treatment failure).[4] Bunin et al. performed a randomized trial of oral $\mathrm{Bu}$ vs. TBI in children with ALL. There was no significant difference between $\mathrm{Bu}$ and TBI for patients who received stem cells from related donors $(36 \%$ vs $58 \%$ ). However, for unrelated donors, EFS was $20 \%$ for $\mathrm{Bu}$ and $57 \%$ for TBI. Relapse was similar in both arms. [5] The available reports did not clarify whether all allogenic stem cell transplant patients need a TBI containing regimen. $[14,15]$

In conclusion, TBI has been used most frequently for allogenic transplantation in patients with acute leukaemia before HSCT. The main drawbacks of allogenic transplantation are early transplant-related mortality and late complications with the latter impacting both quality of life and patient outcomes. At present, there are a lot of unanswered questions about TBI techniques, indications and dose. Developing national protocols will improve TBI procedures in our country.

\section{Disclosure Statement}

The authors declare no conflicts of interest.

\section{References}

1. Walker T, Milford E, Chell J, Maiers M, Confer D. The National Marrow Donor Program: improving access to hematopoietic cell transplantation. Clin Transpl 2011:55-62.

2. Altschuler C, Resbeut M, Blaise D, Maraninchi D, Stoppa AM, Lagrange JL, et al. Fractionated total body irradiation and bone marrow transplantation in acute lymphoblastic leukemia. Int J Radiat Oncol Biol Phys 1990;19(5):1151-4.

3. Aur RJ, Pinkel D. Total therapy of acute lymphocytic leukemia. Prog Clin Cancer 1973;5:155-70.

4. Davies SM, Ramsay NK, Klein JP, Weisdorf DJ, Bolwell $\mathrm{B}$, Cahn JY, et al. Comparison of preparative regimens in transplants for children with acute lymphoblastic leukemia. J Clin Oncol 2000;18(2):340-7.

5. Bunin N, Aplenc R, Kamani N, Shaw K, Cnaan A,
Simms S. Randomized trial of busulfan vs total body irradiation containing conditioning regimens for children with acute lymphoblastic leukemia: a Pediatric Blood and Marrow Transplant Consortium study. Bone Marrow Transplant 2003;32(6):543-8.

6. Shi-Xia X, Xian-Hua T, Hai-Qin X, Bo F, Xiang-Feng T. Total body irradiation plus cyclophosphamide versus busulphan with cyclophosphamide as conditioning regimen for patients with leukemia undergoing allogeneic stem cell transplantation: a meta-analysis. Leuk Lymphoma 2010;51(1):50-60.

7. Gruen A, Ebell W, Wlodarczyk W, Neumann O, Kuehl JS, Stromberger C, et al. Total Body Irradiation (TBI) using Helical Tomotherapy in children and young adults undergoing stem cell transplantation. Radiat Oncol 2013;8:92.

8. Corvò R, Lamparelli T, Bruno B, Barra S, Van Lint MT, Vitale $\mathrm{V}$, et al. Low-dose fractionated total body irradiation (TBI) adversely affects prognosis of patients with leukemia receiving an HLA-matched allogeneic bone marrow transplant from an unrelated donor (UDBMT). Bone Marrow Transplant 2002;30(11):717-23.

9. Springer A, Hammer J, Winkler E, Track C, Huppert $\mathrm{R}, \mathrm{Böhm} \mathrm{A}$, et al. Total body irradiation with volumetric modulated arc therapy: Dosimetric data and first clinical experience. Radiat Oncol 2016;11:46.

10. Kim TH, McGlave PB, Ramsay N, Woods W, Bostrom B, Vercellotti G, et al. Comparison of two total body irradiation regimens in allogeneic bone marrow transplantation for acute non-lymphoblastic leukemia in first remission. Int J Radiat Oncol Biol Phys 1990;19(4):889-97.

11. Clift RA, Buckner CD, Appelbaum FR, Bearman SI, Petersen FB, Fisher LD, et al. Allogeneic marrow transplantation in patients with acute myeloid leukemia in first remission: a randomized trial of two irradiation regimens. Blood 1990;76(9):1867-71.

12. Vriesendorp HM, Herman MG, Saral R. Future analyses of total body irradiation. Int J Radiat Oncol Biol Phys 1991;20(3):635-7.

13. Kal HB, Loes van Kempen-Harteveld M, Heijenbrok-Kal MH, Struikmans H. Biologically effective dose in total-body irradiation and hematopoietic stem cell transplantation. Strahlenther Onkol 2006;182(11):672-9.

14. Mohty M, Malard F, Savani BN. High-dose total body irradiation and myeloablative conditioning before allogeneic hematopoietic cell transplantation: time to re- 
think? Biol Blood Marrow Transplant 2015;21(4):620-4 15. Park J, Choi EK, Kim JH, Lee SW, Song SY, Yoon SM, et al. Effects of total body irradiation-based condition- ing on allogeneic stem cell transplantation for pediatric acute leukemia: a single-institution study. Radiat Oncol J 2014;32(3):198-207. 